\title{
The capillary bistable switch constrained by pinning/wetting angles as a sensor of pressure
}

\author{
Bartłomiej Hanysz and Waldemar Nowicki ${ }^{\mathrm{a}}$ \\ Faculty of Chemistry, Adam Mickiewicz University in Poznań, Umultowska 89b, 61-614 Poznań, Poland
}

Received 12 June 2019 and Received in final form 30 June 2019

Published online: 2 September 2019

(c) The Author(s) 2019. This article is published with open access at Springerlink.com

\begin{abstract}
A droplet deposited onto an orifice in the flat smooth plate can remain in a bistable state. Such system - a droplet and a flat surface - behaves as a bistable capillary switch, which can be used for threshold detection of some system's properties, e.g., the difference in the pressure on both sides of the plate. The droplet morphology changes abruptly at a certain value of the pressure difference and shows hysteresis. The specific behavior of the system is a result of the geometrical constraints defining the curvature of the liquid droplet at both sides of the surface. These constraints can be represented either by pinning angles at both sides of the plate or by the pinning angle at one side and the contact angle at the other. The dependence of details of the droplet morphology and energy on the difference in pressure at both sides of the plate is calculated by means of the semi-analytical model and Surface Evolver simulations.
\end{abstract}

\section{Introduction}

A capillary switch is a bistable system of liquid/gas or immiscible liquid/liquid interfaces with a trigger to toggle back and forth between the two or more stable equilibrium states [1]. To switch from one stable state to the other, the energy barrier must be overcome. The energy barrier can be tuned by the droplet and surrounding morphology. A capillary system becomes a real switch only when toggling is achieved [2]. The toggling trigger can be based on the pressure difference [3], liquid surface tension, inertia force, electric field (e.g., when the electrostatic potential acts to oxidize the surfactant on the one part of the droplet surface and to reduce it on the other part) [3], magnetic field (applied to ferrofluids) [4,5], etc. The droplet properties determining the behavior of the capillary switch include the liquid's physical properties (e.g., zeta potential, surface tension and contact angles) [6,7]. A droplet realizing the bistable switch shows the unusual bifurcation dependence of the droplet morphology on its volume and hence at a volume large enough the morphology vs. stimulant dependence forms a hysteresis loop. The geometrical constraints of the morphology of the liquid surface usually are reduced to the pinning angle of the orifice/pipe edges. However, there are systems in which the motion possibilities of the triple line can be switched from the free shift on the solid surface to the immobilization on linear inhomogeneities (edges) as found for the vertically submerged cylinder [8], the liquid between fiber and substrate [9] and

\footnotetext{
a e-mail: gwnow@amu.edu.pl
}

in Wenzel or Cassie wetting regimes [10]. Moreover, the viscous dissipation by the movement of the triple-contact line, where a fluid/fluid interface meets a solid is also discussed; e.g., Lea [11] demonstrated a bistable switch in geometries with the moving contact line, which is dissipative [12].

The main aim of the study is to answer the question whether the morphology of the surface of the liquid drop, constituting the capillary switch, depends on details of the solid at the site at which the droplet is settled (flat plate, outlet of tube). Namely, the surface morphologies determined by the pinning angle and contact angle are studied. Sections 2 and 3 contain the semi-analytical and simulation approaches to the problem. In sect. 3 , the influence of the gravitational field on the liquid morphology is analyzed. The obtained results are discussed in terms of adjusting and calibration of bistable switches based on different constraints of droplet settlement in practical applications.

\section{The effect of wettability details on the droplet morphology - a semi-analytical approach}

In order to analyze the influence of geometry constraints on the behavior of the capillary switch, let us consider the simplified system in which a droplet is settled on the orifice of radius $R_{0}$ in the flat smooth plate with negligibly small thickness. Additionally let us assume that the 


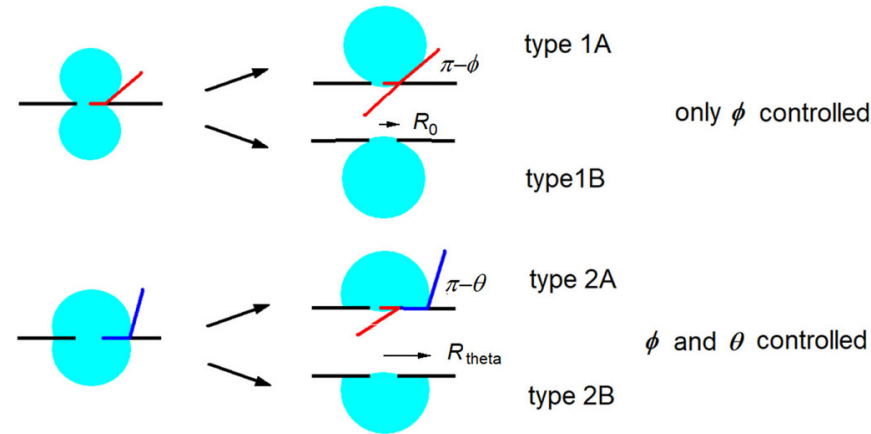

a)

b)

Fig. 1. The unstable symmetrical initial droplet morphology at the zero pressure difference (a) and possible equilibrium states of type 1A and 1B (controlled by the pinning angle $\phi$ marked in red) and type $2 \mathrm{~A}$ and $2 \mathrm{~B}$ (controlled by the pinning angle $\phi$ marked in red on one side of the plate and by the contact angle $\theta$ marked in blue on the other side) (b). For unification of values of contact and pinning angle it is assumed that the horizontal shoulder of the pinning angle does not lie on the surface but it overhangs the surface, thus the actual pinning angle is $\pi-\phi$.

droplet is small enough for the system to be in the pure capillary regime (the Bond number $B o \ll 1[13]$ ). In such case, both droplet surfaces form spherical caps of the mean curvature radii equal to $R_{1}$ and $R_{2}$, respectively. If the gas pressure is the same on both sides of the plate, both cap radii take the same value $\left(R_{1}=R_{2}\right)$ as a result of the Laplace law [14]. However, the symmetrical morphology of two identical spherical caps (see fig. 1(a)) is metastable and tends to one of the two equilibria states of smaller surface/interface energy (fig. 1(b)) with the same probability. Thus, the system's evolution shows bifurcation. The initial symmetrical state as well as the resulting equilibria states depend on the plate surfaces wettability and can be governed by the pinning or contact angles as shown in fig. 1 .

Let us notice that the droplet morphology of type $1 \mathrm{~A}$ and $1 \mathrm{~B}$ is determined by the pinning effect of the droplet surface to the edge of the orifice. As a consequence, the angles between the tangent to the liquid surface and solid surface $\phi$ can vary in the way fulfilling the inequality

$$
2 \pi-\theta \leq \phi \leq \theta
$$

The corresponding angles between the surfaces in type $2 \mathrm{~A}$ and $2 \mathrm{~B}$ can either take the value limited by inequality (1) or should be strictly determined by the contact angle $\theta$.

Changes in the pressure difference on both sides of the plate can affect the curvature radii of both spherical caps according to the Laplace law

$$
\begin{aligned}
& p-p_{1}=\frac{2 \gamma}{R_{1}}, \\
& p-p_{2}=\frac{2 \gamma}{R_{2}}
\end{aligned}
$$

where $p_{1}, p_{2}$ and $p$ are pressures at both sides of the plate and the internal pressure in the droplet, respectively. Hence, the difference in pressures defines the difference in curvature of both spherical caps

$$
\Delta p=p_{1}-p_{2}=2 \gamma\left(\frac{1}{R_{1}}-\frac{1}{R_{2}}\right) .
$$

For the upper and lower pinned cups of type 1A or $1 \mathrm{~B}$ constrains, the curvature radius can be related to the orifice radius

$$
\begin{aligned}
& R_{1}=\frac{R_{0}}{\sin \phi_{1}}=\frac{A R}{\sin \phi_{1}}, \\
& R_{2}=\frac{R_{0}}{\sin \phi_{2}}=\frac{A R}{\sin \phi_{2}},
\end{aligned}
$$

where $A$ is the aspect ratio equal to the ratio of $R_{0}$ to $R=(3 V /(4 \pi))^{1 / 3}$ where $V$ is the droplet volume.

In the case of type $2 \mathrm{~A}$ constrain, eq. (5) is still valid but, in eq. (6), the orifice radius should be replaced by the radius of droplet bottom at the constant contact angle $\theta$ :

$$
R_{2}=\frac{R_{\text {theta }}}{\sin \theta}
$$

where $R_{\text {theta }}$ is the radius of the triple line circle.

By combining eqs. (4)-(7), the pinning angle $\phi_{1}$ can be related to $\Delta p$ by

$$
\frac{\sin \phi_{1}}{A R}=\frac{\sin \phi_{2}}{A R}+\frac{\Delta p}{2 \gamma}
$$

for type 1 (when $\Delta p$ changes, $R_{0}=A R$ remains constant), and

$$
\frac{\sin \phi_{1}}{R_{\text {theta }}}=\frac{\sin \theta}{A R}+\frac{\Delta p}{2 \gamma},
$$

for type 2 (when $\Delta p$ changes, $\theta$ remains constant).

The sum of the volumes of both caps is equal to the volume of the whole droplet $V$,

$$
V=V_{1}+V_{2},
$$

and the volume of a single spherical cap reads

$$
V_{x}=\frac{\pi}{3}\left(\frac{R_{x}}{\sin \alpha}\right)^{3} F(\alpha),
$$

where

$$
F(\alpha)=\cos ^{3} \alpha-3 \cos \alpha+2,
$$

where $R_{x}$ is the radius of the cup basis equal to $A R$ (pinned cup) or $R_{\text {theta }}$ (wetting cup) and $\alpha$ is the pinning or contact angle.

The total volume of the droplet is equal to

$$
\begin{aligned}
V= & \frac{\pi(A R)^{3}}{3}\left[\frac{1}{\sin ^{3} \phi_{1}} F\left(\phi_{1}\right)+\frac{1}{\left(\sin \phi_{1}-\frac{A R \Delta p}{2 \gamma}\right)^{3}}\right. \\
& \left.\times F\left(\arcsin \left(\sin \phi_{1}-\frac{A R \Delta p}{2 \gamma}\right)\right)\right]
\end{aligned}
$$




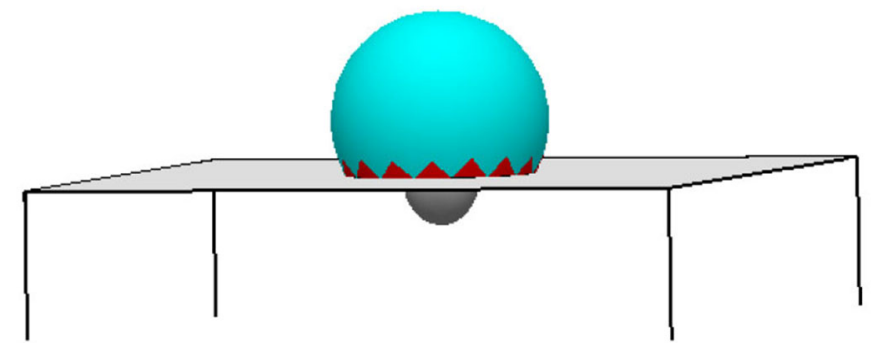

Fig. 2. The visualization of the droplet of morphology controlled by contact angle (top) and pinning angle (bottom) (type $2 \mathrm{~A}$ ). Triangles used to calculate the pinning/contact angle $\alpha$ are marked in red.

in the case of type 1 constraint, and

$$
V=\frac{\pi(A R)^{3}}{3}\left[\frac{1}{\sin ^{3} \phi_{1}} F\left(\phi_{1}\right)+\frac{1}{\left(\sin \phi_{1}-\frac{A R \Delta p}{2 \gamma}\right)^{3}} F(\theta)\right],
$$

for type 2 constraint.

Analysis of eq. (8) indicates that the expression governing the variability of pinning angle is $\Delta p A / \gamma$ hence one can expect that $A$ and $1 / \gamma$ will linearly influence the effect of $\Delta p$. Let us also notice that, in the wide range of applied $\Delta p$, one of the components of total volume of the droplet, $V_{1}$ or $V_{2}$, can take negative values when the surface bends in the direction opposite to that shown in fig. 1.

A comparison of eqs. (13) and (14) shows that they are almost identical except for the difference in the argument in the $F$ function addressed to one of the cups, which raises the suspicion that the behavior of systems of type 1 and 2 can differ.

Equations (13) and (14) can be treated as $\phi_{1}=f(\Delta p)$ functions. However, the functions are implicit and can be solved only by numerical methods. This solution leads also to determination of all geometric parameters of droplet morphology based on the equation set (2)-(9). However, because of the high number of roots of eqs. (13) and (14), it is quite difficult to calculate the parameters of interest and in several cases the obtained results were doubtful. Moreover, the solution is limited to the non-gravity systems. In such case, the approximate calculations made for systems under gravity with the finite element method seem to give less precise but more reliable results.

\section{The capillary switch — the finite element method simulation}

This part of the study was performed by means of simulations with the finite element method using the Surface Evolver (SE) program $[15,16]$. The surface modeled by SE is represented by a mesh network of triangles whose vertices make the network nodes. The system studied was a droplet settled on the plate of negligibly small thickness (see fig. 2). The study was performed for the cup edges forced to be pinned to the orifice edge (type $1 \mathrm{~A}$ and $1 \mathrm{~B})$ and for the cup edges freely moving on the surface (type $2 \mathrm{~A}$ and $2 \mathrm{~B}$ ). In the latter case, the pinning effect can be caused spontaneously by the system geometry.
Each simulation started with the two equilibrated cups placed symmetrically at both sides of the plate around the orifice at $\Delta p=0$. Then the initial value of $\Delta p$ was applied and the droplet morphology was immediately switched to the asymmetrical state of minimum energy, further modified by subsequent pressure changes.

In the course of simulation, $\Delta p$ was changed step by step by the value of $20 \mathrm{~Pa}$ (or $5 \mathrm{~Pa}$ when gravity was switched on). Then the free energy of the modelled system was minimized in defined steps including the procedures of mesh refinement, vertex averaging, polishing up the triangulation and the energy minimization by means of the conjugated gradient descent method. The range of $\Delta p$ studied depended on the system parameters (up to $-10^{3}-10^{3} \mathrm{~Pa}$ ). The other parameters: the droplet volume $V=1 \cdot 10^{-9} \mathrm{~m}^{3}$, the liquid surface tension $\gamma=0.072 \mathrm{~J} / \mathrm{m}^{2}$, and liquid density $\rho=1 \cdot 10^{3} \mathrm{~kg} / \mathrm{m}^{3}\left(\mathrm{H}_{2} \mathrm{O}\right)$ were kept constant. Separate simulations were performed for different values of the droplet volume at the acceleration due to gravity of $9.81 \mathrm{~m} / \mathrm{s}^{2}$, the contact angle $\theta=120^{\circ}$ and aspect ratio $A=0.3$.

The values of the pinning/contact angle $\alpha$ were calculated on the basis of inclination of the mesh triangles representing the free liquid surface and being in contact with the triple line to the plane of the solid surface (see fig. 2), from the equation

$$
\alpha=\arccos \left(\left\langle\frac{z}{S_{\mathrm{T}}}\right\rangle\right),
$$

where $z$ and $S_{\mathrm{T}}$ denote the vertical coordinate and the length of the normal vector of each triangle used for calculation of the surface slope near the triple line, marked in red in fig. 2 (the length $S_{\mathrm{T}}$ of $\mathrm{SE}$ representation of the normal vector is not unitary but equals to the area of the triangle).

The exemplary evolution of the contact/pinning angle (denoted as $\alpha$ ) on both sides of the plate in the course of one and a half cycle of changes in $\Delta p$ at zero gravity, is shown in fig. 3 . Both curves are symmetrical -when, on the one side of the plate, only the variation in the size of the cup is observed (at constant contact angle), on the other side, changes in the shape of the cup occur with varying the pinning angle. The unsymmetrical line in the figure refers to the starting steps of simulation. Slightly inflated values of $\alpha$ presented in fig. 3 and the next drawings are a result of the assumed method of calculation and are related to the fact that the triangles used for the calculation of the liquid surface slope have finite size. The width of the loops is limited by two critical pressure differences switching the droplet morphology from one to the other $\Delta p_{\text {crit_I }}$ and $\Delta p_{\text {crit_II }}$.

The influence of the aspect ratio on the $\alpha$ variation for the droplet located on a plate with the contact angle $\theta=120^{\circ}$ in both types of pinning/wetting phenomena is shown in fig. 4. All hysteresis curves presented are similar to that shown in fig. 3 and can be interpreted in the same way, thus for simplicity only the dependencies of $\alpha_{1}$ (further referred as $\alpha$ ) on $\Delta p$ at the single side of the plate are shown. As can be seen, the curves obtained for 


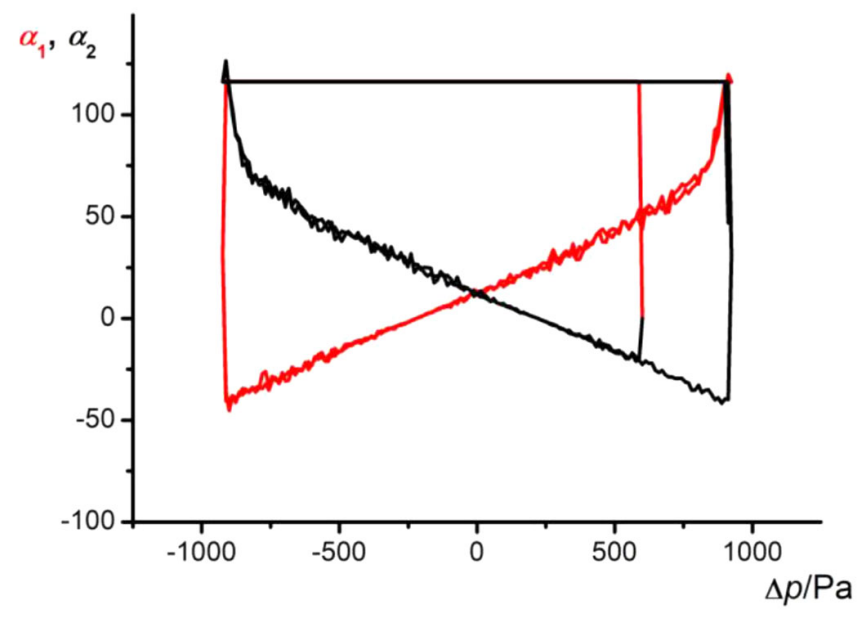

Fig. 3. The dependence of $\alpha$ of both cups on the pressure difference (type $2, \theta=120^{\circ}$ ). The $\alpha_{1}=f(\Delta p)$ dependence is partly hidden by the following relationship $\alpha_{2}=f(\Delta p)$.

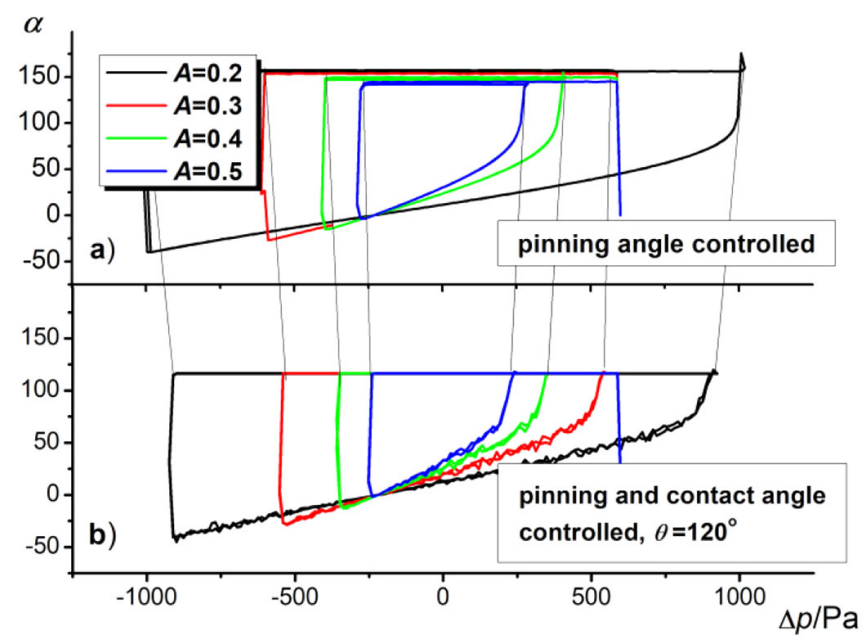

Fig. 4. Evolution of the pinning/contact angle $\alpha$ caused by changes in pressure difference: (a) in the system where the droplet is pinned to edge of the orifice (type 1) and (b) where droplet partly wets the surface with the contact angle $120^{\circ}$ (type 2). The lines between both parts of the figure connect the points of morphology switching.

type 1 constraint slightly differ from that for type 2 . The difference concerns the $\alpha$ angle at the side of the plate at which the liquid volume is currently larger: for half of the cycle of pressure changes, the $\alpha$ angle for the type 1 constraint tries to reach a very high value of about $\alpha=160^{\circ}$, whereas for the type 2 constraint, $\alpha=\theta$. For another half of the cycle, the $\alpha$ angle is not constant but limited by the pining requirements (inequality 1 ) and takes smaller values. At the same time $\alpha_{2}=160^{\circ}$ (type 1) or $\alpha_{2}=\theta$ (type 2) on the other side of the plate. In the first case $\left(\alpha \approx 160^{\circ}\right)$, in the real experiment the spreading of the droplet over the plate with $\theta=120^{\circ}$ should be observed (type 2) instead of the increase in $\alpha$ to a very high value $\left(160^{\circ}\right)$. However, for selected geometries of the solid surface when inequality (1) can be still fulfilled (on the edge of the thin-walled tube instead of a flat plate), one can expect the $\alpha=f(\Delta p)$ shown in fig. 4 .

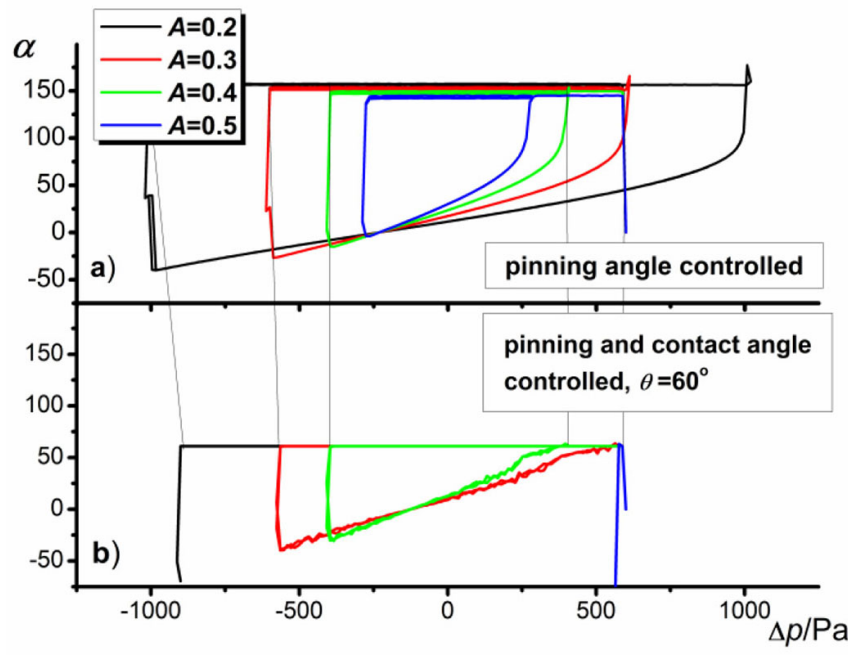

Fig. 5. Evolution of the pinning/contact angle $\alpha$ caused by changes in pressure difference: (a) in the system where the droplet is pinned to edge of the orifice (type 1) and (b) where droplet partly wets the surface with the contact angle $60^{\circ}$ (type 2). The lines between both parts of the figure connect the morphology switching points.

The bistable switches based on the different constraints represented by type 1 and 2 work differently - the maximum volume of the liquid droplet switched to the equilibrium state (eq. (11)) is different in both cases, so the detection method should be precisely adjusted to the type of constraint. More important, however, is the fact that the threshold pressures at which the droplet morphology switches from one to the other $\left(\Delta p_{\text {crit_I }}\right.$ and $\left.\Delta p_{\text {crit_II }}\right)$ in both types of constrains also differ from each other as shown by the lines connecting points of switching morphology in figs. 4(a) and (b). The range of $\Delta p$ not causing a jump in droplet morphology (the plateau region) is wider in the type 1 constraint.

Figure 5 shows the influence of the aspect ratio on the $\alpha$ variation for the droplet located on a plate with the contact angle $\theta=60^{\circ}$. The number of available results is significantly smaller (especially for type 2 constraint) as a result of relatively poor convergence of calculations. However, both curves families (for type 1 and 2 constraints) demonstrate analogous properties and indicate the same problems with adjusting and calibrating of bistable switches of both types.

\section{The capillary switch under gravity}

A large drop in the presence of gravity (for relatively large Bond numbers $(B o>1))$, defined by the equation

$$
B o=\frac{\rho g V^{\frac{2}{3}}}{\gamma},
$$

is unstable, detaches itself from the plate and falls down (e.g. for $V=30 \mathrm{~mm}^{3}, B o=1.316$ ). The behavior of capillary switches based on smaller droplets is dependent on the droplet volume. Figure 6 shows the $\alpha$ angle hysteresis loops obtained for two droplets of the volume $V=5$ and 


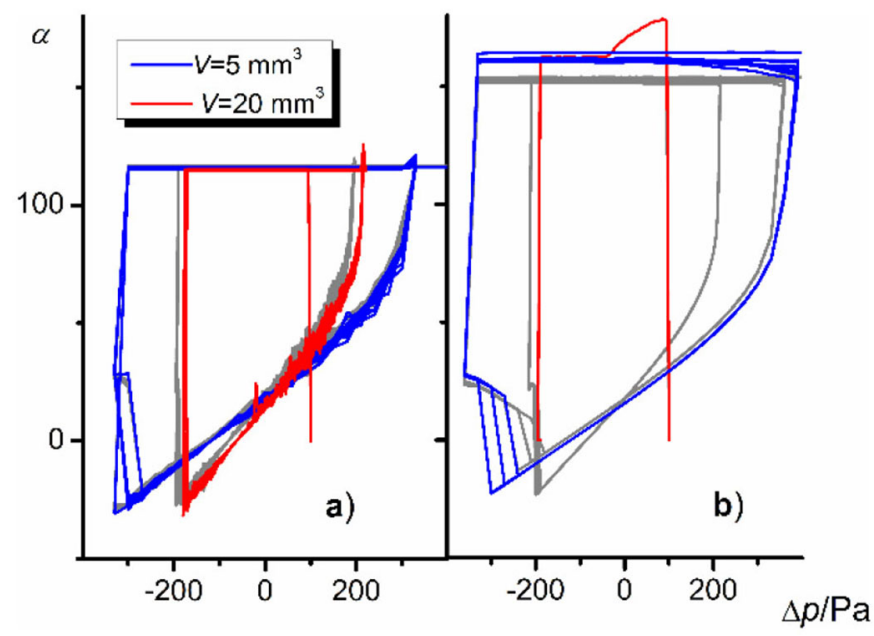

Fig. 6. Evolution of the pinning/contact angle $\alpha$ caused by changes in pressure difference in the system: (a) controlled by pinning and contact angle, (b) controlled by pinning $\left(\theta=120^{\circ}\right.$, $A=0.3, g=9.81 \mathrm{~m} / \mathrm{s}^{2}$, the droplet volumes are indicated in the figure). For comparison, the loops obtained in the absence of gravitational field are also shown in gray.

$20 \mathrm{~mm}^{3}$ (Bo $=0.398$ and 1.004 , respectively). The dependencies were obtained at the same aspect ratio $A=0.3$. As shown at $V=5 \mathrm{~mm}^{3}$, the gravity practically does not influence the shape of the loop of type 1 and lowers the high critical switching pressure $\left(\Delta p_{\text {crit_II }}\right)$ of type 2 . For a volume very close to $B o=1$, both loops are apparently affected - the regular decrease in both critical switching pressures $\left(\Delta p_{\text {crit_I }}\right.$ and $\left.\Delta p_{\text {crit_II }}\right)$ is observed for type 1 , whereas the dependence for type 2 shows unstable behavior of the droplet and its detachment from the plate.

\section{Conclusions}

The semi-analytical method as well as SE simulation were used to study the behavior of the droplet settled on the orifice in the flat plate at different constraints imposed on the droplet edge - the immobile pinned edge (type 1) or the freely moving triple line at constant contact angle (type 2 ). The methods indicate that droplets of both types behaves similarly - they abruptly switch their morphologies at certain differences in pressure $\Delta p$ at both sides of the plate. However, the angle at the triple line $\alpha$ in the systems of both types differs and the critical switching pressures for all studied aspect ratio are different. Consequently, one can state that the operation of the switch is highly dependent on the precision of the orifice fabrication especially on the sharpness of its edge. Rounded or damaged edges can facilitate detachment of the cup edges and wetting of the surface (equivalent to transition from type 1 to 2 ).
Capillary switches can work even at high wettability of the surface for which the droplet constraint of type 2 are applicable.

The gravitation influences both critical switching pressures $\Delta p_{\text {crit }}$ and shifts the hysteresis loop along the pressure difference axis.

\section{Author contribution statement}

Both authors contributed equally to this work.

Publisher's Note The EPJ Publishers remain neutral with regard to jurisdictional claims in published maps and institutional affiliations.

Open Access This is an open access article distributed under the terms of the Creative Commons Attribution License (http://creativecommons.org/licenses/by/4.0), which permits unrestricted use, distribution, and reproduction in any medium, provided the original work is properly cited.

\section{References}

1. D.P.J. Bartz, P.H. Steen, Phys. Fluids 25, 097104 (2013).

2. H.C. Wente, Pac. J. Math. 189, 339 (1999).

3. A.H. Hirsa, C.A. Lopez, M.A. Laytin, Appl. Phys. Lett. 86, 014106 (2005).

4. B.A. Malouin jr., M.J. Fogel, J.D. Olles, L. Cheng, A.H. Hirsa, Lab Chip 11, 393 (2011).

5. M.J. Vogel, P. Ehrhard, P.H. Steen, Proc. Natl. Acad. Sci. U.S.A. 102, 11974 (2005).

6. J. Xie, J. Shih, Q. Lin, B. Yang, Y.-C. Tai, Lab Chip 4, 495 (2004)

7. L.X. Chen, J.P. Ma, F. Tan, Y.F. Guan, Sens. Actuators B 88, 260 (2003).

8. J. Liu, J. Sun, Y. Mei, Appl. Phys. Lett. 104, 231607 (2014).

9. J. Qian, H. Gao, J. Acta Biomater. 2, 51 (2006).

10. J. Liu, Y. Mei, R. Xia, Langmuir 27, 196 (2011).

11. M.C. Lea, J. Appl. Phys. 58, 108 (1985).

12. P.G. de Gennes, Rev. Mod. Phys. 57, 827 (1985).

13. W.H. Hager, J. Hydraul. Res. 50, 3 (2012).

14. J. Berthier, K.A. Brakke, The Physics of Microdroplets (John Wiley \& Sons, Inc. Hoboken, NJ, 2012).

15. K.A. Brakke, Exp. Math. 1, 141 (1992).

16. K.A. Brakke, Surface Evolver Manual, Version 2.70 (2014) http://www. susqu.edu/brakke/evolver/html/evolver. htm. 